Research Article

\title{
Effectiveness of Intracanal Cryotherapy using Different Irrigants on Reduction of Post Endodontic Pain - A Systematic Review and Meta-analysis
}

\author{
${ }^{*}$ Dr. Parekh Janhvi S, ${ }^{1}$ Dr. Hegde Vibha R (MDS), ${ }^{2}$ Dr. Vaidya Mrunalini J (MDS) \\ *Department of Conservative Dentistry and Endodontics, Dr. G. D. Pol Foundation's YMT Dental College, Navi Mumbai, India. \\ ${ }^{1}$ Professor and Head of Department of Conservative Dentistry and Endodontics, Dr. G. D. Pol Foundation's YMT Dental College, Navi Mumbai, India \\ ${ }^{2}$ Professor, Department of Conservative Dentistry and Endodontics, Dr. G. D. Pol Foundation's YMT Dental College, Navi Mumbai, India. \\ *Corresponding author's E-mail: janhviparekhjp@gmail.com
}

Received: 03-11-2021; Revised: 24-01-2022; Accepted: 05-02-2022; Published on: 15-02-2022.

\begin{abstract}
Introduction: Recently the non-pharmacological management of post endodontic pain has been extensively studied. The aim of this systematic review was to evaluate the effectiveness of intracanal cryotherapy in reducing postoperative endodontic pain. A comprehensive search was conducted by accessing electronic databases like PubMed, Google Scholar, Google and EBSCO. Articles evaluating the effect of intracanal cryotherapy in reducing postoperative pain using different irrigating agents which were published till November 2020 were included in the study. Risk of bias was assessed using the Cochrane risk of bias criteria. Meta-analysis was performed for ten studies and tested the heterogeneity using 12 index. Qualitative and quantitative analysis was done for thirteen and ten studies respectively. Intracanal cryotherapy was found to be effective in reducing post-operative pain at 6 hours and 24 hours. Within the limitations of the study, it can be concluded that intracanal cryotherapy using cold saline or $17 \%$ EDTA at a temperature range of $2.5^{\circ} \mathrm{C}$ to $6^{\circ} \mathrm{C}$ as final irrigating agent helps in reducing postoperative pain at $6 \mathrm{hrs}$ and $24 \mathrm{hrs}$ following single-visit or multi visit root canal treatment in teeth diagnosed with irreversible pulpitis with apical periodontitis.
\end{abstract}

Keywords: Intracanal cryotherapy, cold saline, postoperative pain.

QUICK RESPONSE CODE $\rightarrow$

DOI:

10.47583/ijpsrr.2022.v72i02.022

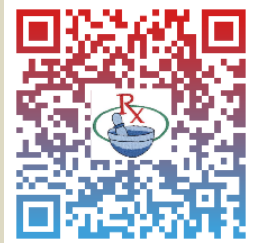

DOI link: http://dx.doi.org/10.47583/ijpsrr.2022.v72i02.022

\section{INTRODUCTION}

ostendodontic pain is multifactorial ${ }^{1}$ and is linked to periapical inflammatory response which could be secondary to anatomical, mechanical, chemical and/or microbial factors associated with the tooth or the periradicular tissues ${ }^{2-5}$. The non-pharmacological methods used to mitigate postoperative pain include counselling, preoperative patient-calming approaches and explanations $^{6}$, occlusal reductions ${ }^{7}$, verbal anesthesia, music therapy, medication to more specific counselling and operative procedures like cryotherapy. The pharmacologic methods include, long-acting anesthesia, medication using antihistamines ${ }^{8}$, nonsteroidal antiinflammatory drugs $^{9}$, narcotic analgesics, and steroidal anti-inflammatory drugs ${ }^{10}$. Moreover, the use of medications to relieve pain is associated with an increased risk of harm, including gastrointestinal irritation and other systemic adverse effects ${ }^{11}$. Thus, alternative techniques such as cryotherapy are being evaluated for management of postoperative pain.

Cryotherapy is a relatively new form of treatment which involves applying cold through various methods which may decrease the conduction velocity of nerve signals, reduces the local blood flow by vasoconstriction and hence reduce hemorrhage, edema and local inflammation ${ }^{11,12}$ in order to promote healing and other therapeutic results. One way to apply cryotherapy to the inflamed periradicular tissues is by intracanal irrigation with a cold substance after the endodontic cleaning and shaping procedure. Number of studies ${ }^{13-15}$ have been performed to evaluate the effectiveness of intracanal cryotherapy in reducing the postoperative pain, however, a systematic analysis of the quality of these randomized controlled trials needs to be evaluated using well defined criterias like those used in a systematic review. Thus, the aim of this systematic review was to evaluate the effectiveness of intracanal cryotherapy in reducing postoperative endodontic pain.

\section{MATERIALS AND METHODS}

\section{Protocol and registration}

The research protocol is designed according to the PRISMA (Preferred Reporting Items for Systematic Review and Meta-Analyses) guidelines 2009. The protocol of this systematic review was registered with the International Prospective Register of Systematic Review (PROSPERO CRD42021229572).

\section{Focused question}

Is intracanal cryotherapy (intervention) effective in decreasing the postoperative pain (outcome) as compared to the use of irrigants at room temperature (comparator) after cleaning and shaping in endodontic treatment in adult permanent teeth (population)? 


\section{PICOS criteria}

POPULATION: Adults permanent teeth undergoing endodontic treatment

\section{INTERVENTION: Cryotherapy}

COMPARISON: Saline at room temperature

OUTCOME: Post-operative pain

STUDY DESIGN: RCTS

\section{Eligibility criteria}

\section{Inclusion Criteria}

The inclusion criteria included studies which employed the following:

- patients of varied age groups requiring endodontic treatment of permanent teeth

- irrigants at different temperatures as treatment modalities for endodontic pain reduction

- minimum follow up of up to 24 hours

- multiple techniques of irrigation for control and study group of cryotherapy

- Publications in English, with full text available in either soft or hard copy.

\section{Exclusion Criteria}

The exclusion criteria included the studies which employed the following:

- $\quad$ use of different techniques to reduce post endodontic pain

- $\quad$ multiple dependent variables besides post endodontic pain

- multiple independent variables besides cryotherapy

- Non clinical treatment (eg, studies on cell cultures or animal study).

- Publications were in the form of letters, commentaries, or narratives.

- No specified criteria provided for evaluating the outcome of treatment, or no mention of how to determine the healing outcome.

\section{Literature search}

A comprehensive search was conducted by accessing electronic databases, along with a manual search, to identify all relevant studies associated with intracanal cryotherapy. Electronic databases, like PubMed, Google Scholar, Google and EBSCO were consulted by looking for the MeSH terms and key words like post endodontic pain, postoperative pain, root canal, irrigation for root canal, cold irrigation, endodontic irrigation, cryotherapy, and intracanal cryotherapy with appropriate Boolean characters.

The search lined all articles printed from 1990 to 2020. Articles from 2016 to 2020 were selected for the study. Duplicate records were removed. Each prospective and retrospective clinical study printed in English language were enclosed. The workflow followed the PRISMA checklist.

All studies known were screened by reading the title and the abstract by two independent reviewers and by applying the inclusion and exclusion criteria. Then full texts of these studies were obtained. Inter-reviewer reliability was assessed with Cohen kappa (0.80). Any possible discrepancies encountered during this process, were resolved by discussion between the reviewers and if a disagreement persisted, the judgment of a third reviewer was considered decisive.

\section{Data collection}

Characteristics of included trials and numerical data were extracted by two reviewers using predetermined and piloted extraction forms. During the protocol stage piloting of the forms was performed until over $90 \%$ agreement was reached.

\section{Data extraction and data items}

Information on authors' names, year of publications, study design, sample size, number of visits, preoperative pulpal and periapical diagnosis, type of teeth included, groups of intervention, follow-up period, method of irrigation, volume of irrigation, temperature of the irrigant, method of pain assessment and the result was independently extracted by two reviewers. Data regarding the included studies was also independently extracted by the reviewers based on a previously defined protocol in a specific form in the Microsoft Office Excel 2007 software (Microsoft Corporation, Redmond, WA, USA) [Table 1].

Table 1: PICOS Data Extraction Chart

\begin{tabular}{|c|c|c|c|c|c|c|c|c|c|}
\hline Sr No & Author (Year) & $\begin{array}{l}\text { Sample } \\
\text { size }\end{array}$ & $\begin{array}{l}\text { No. of } \\
\text { visit }\end{array}$ & $\begin{array}{l}\text { Type of tooth } \\
\text { /Diagnosis }\end{array}$ & $\begin{array}{c}\text { method of } \\
\text { irrigation used }\end{array}$ & $\begin{array}{l}\text { Final irrigation } \\
\text { regimen in } \\
\text { experimental group }\end{array}$ & $\begin{array}{l}\text { Final irrigation } \\
\text { regimen in } \\
\text { Control group }\end{array}$ & Results & $\begin{array}{l}\text { follow up } \\
\text { (time } \\
\text { interval) }\end{array}$ \\
\hline 1 & $\begin{array}{l}\text { Al-Nahlawi et } \\
\text { al. }(2016)^{13}\end{array}$ & 75 & $\begin{array}{l}\text { single } \\
\text { visit }\end{array}$ & $\begin{array}{l}\text { Single-rooted - } \\
\text { single canal } \\
\text { teeth with Vital } \\
\text { teeth with } \\
\text { irreversible } \\
\text { pulpitis or pulp } \\
\text { exposure }\end{array}$ & $\begin{array}{c}\text { Endovac } \\
\text { microcannula }\end{array}$ & $\begin{array}{l}\text { Group II: } 20 \mathrm{~mL} \text { of } \\
\text { room temperature } \\
\text { saline was irrigated } \\
\text { for } 5 \text { minutes using } \\
\text { EndoVac }\end{array}$ & $\begin{array}{c}\text { Group I - No } \\
\text { additional } \\
\text { irrigation was } \\
\text { applied (control). }\end{array}$ & $\begin{array}{l}\text { Pain levels were } \\
\text { high ingroups I and } \\
\text { II after } 6 \text { hours that } \\
\text { decreased with time } \\
\text { to almostdiminish } \\
\text { after } 1 \text { week, and on } \\
\text { the other hand, }\end{array}$ & $\begin{array}{l}6,12,24, \\
48 \text { hours, } \\
\text { and } 7 \text { days }\end{array}$ \\
\hline
\end{tabular}




\begin{tabular}{|c|c|c|c|c|c|c|c|c|c|}
\hline Sr No & Author (Year) & $\begin{array}{c}\text { Sample } \\
\text { size }\end{array}$ & $\begin{array}{l}\text { No. of } \\
\text { visit }\end{array}$ & $\begin{array}{l}\text { Type of tooth } \\
\text { /Diagnosis }\end{array}$ & $\begin{array}{c}\text { method of } \\
\text { irrigation used }\end{array}$ & $\begin{array}{l}\text { Final irrigation } \\
\text { regimen in } \\
\text { experimental group }\end{array}$ & $\begin{array}{l}\text { Final irrigation } \\
\text { regimen in } \\
\text { Control group }\end{array}$ & Results & $\begin{array}{l}\text { follow up } \\
\text { (time } \\
\text { interval) }\end{array}$ \\
\hline & & & & & & $\begin{array}{l}\text { Group III: A } 20 \mathrm{~mL} \text { of } \\
2 \text { to } 4^{\circ} \mathrm{C} \text { cold saline } \\
\text { was irrigated for } 5 \\
\text { minutes using } \\
\text { EndoVac }\end{array}$ & & $\begin{array}{c}\text { group III showedno } \\
\text { pain among } \\
\text { different monitoring } \\
\text { periods. } \\
\text { Also pain levels in } \\
\text { groups II were lower } \\
\text { compared with } \\
\text { group I after only } 6 \\
\text { hours }\end{array}$ & \\
\hline 2 & $\begin{array}{l}\text { Keskin et al. } \\
(2016)^{14}\end{array}$ & 170 & $\begin{array}{l}\text { single } \\
\text { visit }\end{array}$ & $\begin{array}{l}\text { incisors, } \\
\text { premolars, } \\
\text { molars with } \\
\text { asymptomatic } \\
\text { or symptomatic } \\
\text { irreversible } \\
\text { pulpitis with } \\
\text { either normal } \\
\text { apical tissues or } \\
\text { symptomatic } \\
\text { apical } \\
\text { periodontitis }\end{array}$ & $\begin{array}{l}31 \text { G Navi-Tip } \\
\text { needle } \\
\text { inserted } 2 \mathrm{~mm} \\
\text { short of the } \\
\text { WL }\end{array}$ & $\begin{array}{l}\text { Cryotherapy group - } \\
5 \mathrm{ml} \text { of } 0.9 \% \\
\text { physiological saline } \\
\text { solution at } 2.5^{\circ} \mathrm{C} \text { was } \\
\text { used for } 5 \text { mins }\end{array}$ & $\begin{array}{l}\text { Control group - } \\
5 \mathrm{ml} \text { of } 0.9 \% \\
\text { physiological } \\
\text { saline solution at } \\
\text { room } \\
\text { temperature was } \\
\text { used for } 5 \text { min }\end{array}$ & $\begin{array}{l}2.5^{\circ} \mathrm{C} \text { cold saline } \\
\text { irrigation as final } \\
\text { irrigant can result in } \\
\text { significant reduction } \\
\text { of post-operative } \\
\text { pain as compared to } \\
\text { control group }\end{array}$ & $\begin{array}{c}24 \text { and } 48 \\
h\end{array}$ \\
\hline 3 & $\begin{array}{l}\text { Vera et al. } \\
(2018)^{15}\end{array}$ & 210 & $\begin{array}{l}\text { multiple } \\
\text { visits }\end{array}$ & $\begin{array}{c}\text { uniradicular } \\
\text { teeth with a } \\
\text { single canal with } \\
\text { necrotic pulp } \\
\text { and symptomatic } \\
\text { apical } \\
\text { periodontitis }\end{array}$ & $\begin{array}{c}\text { Endovac } \\
\text { microcannula }\end{array}$ & $\begin{array}{l}\text { Cryotherapy group - } \\
20 \mathrm{~mL} \text { sterile cold } \\
\left(2.5^{\circ} \mathrm{C}\right) \text { saline } \\
\text { solution is delivered } \\
\text { to the working length } \\
\text { with a sterile, cold } \\
\left(2.5^{\circ} \mathrm{C}\right) \text { Endovac } \\
\text { microcannula for } 5 \\
\text { mins }\end{array}$ & $\begin{array}{l}\text { Control group - } \\
20 \mathrm{~mL} \text { sterile } \\
\text { room temp saline } \\
\text { solution } \\
\text { delivered to the } \\
\text { working length } \\
\text { with a sterile } \\
\text { microcannula } \\
\text { attached to } \\
\text { Endovac for } 5 \\
\text { mins }\end{array}$ & $\begin{array}{c}\text { Patients in the } \\
\text { cryotherapy group } \\
\text { suffered } \\
\text { significantly less } \\
\text { pain after 6, 24, and } \\
72 \text { hours and } \\
\text { needed fewer } \\
\text { analgesics } \\
\text { postoperatively } \\
\text { Patients in the } \\
\text { control group } \\
\text { presented a } \\
\text { significantly higher } \\
\text { incidence of } \\
\text { postoperative pain, } \\
\text { intensity,and need } \\
\text { for medication } \\
\text { intake }\end{array}$ & $\begin{array}{c}6,24 \text {, and } \\
72 \text { hours }\end{array}$ \\
\hline 4 & $\begin{array}{l}\text { Jaiswal et al. } \\
(2020)^{16}\end{array}$ & 30 & $\begin{array}{l}\text { multiple } \\
\text { visits }\end{array}$ & $\begin{array}{l}\text { symptomatic } \\
\text { apical } \\
\text { periodontitis } \\
\text { and pulp } \\
\text { necrosis }\end{array}$ & not mentioned & $\begin{array}{c}\text { Group B } \\
\text { (cryotherapy group) } \\
-20 \mathrm{ml} \text { cold saline } \\
\left(2.5^{\circ} \mathrm{C}\right) \text { is used for } \\
5 \mathrm{mins}\end{array}$ & $\begin{array}{l}\text { Group A ( control } \\
\text { group) - } 20 \mathrm{ml} \\
\text { saline at room } \\
\text { temperature is } \\
\text { used for } 5 \mathrm{mins}\end{array}$ & $\begin{array}{c}\text { No statistical } \\
\text { difference between } \\
\text { the two groups was } \\
\text { seen }\end{array}$ & $\begin{array}{l}6 \text { hours } \\
\text { and } 24 \\
\text { hours }\end{array}$ \\
\hline 5 & $\begin{array}{c}\text { Gundogdu et } \\
\text { al. }(2018)^{17}\end{array}$ & 100 & $\begin{array}{l}\text { single } \\
\text { visit }\end{array}$ & $\begin{array}{c}\text { maxillary or } \\
\text { mandibular } \\
\text { molar with vital } \\
\text { pulp and } \\
\text { symptomatic } \\
\text { apical } \\
\text { periodontitis }\end{array}$ & $\begin{array}{l}\text { needle and } \\
\text { syringe }\end{array}$ & $\begin{array}{l}\text { Intracanal } \\
\text { cryotherapy group - } \\
20 \mathrm{~mL} \text { of cold saline } \\
\text { solution }\left(2.5^{\circ} \mathrm{C}\right) \text { was } \\
\text { used for } 5 \text { minutes. } \\
\text { Intraoral } \\
\text { cryotherapy group - } \\
20 \mathrm{~mL} \text { of room } \\
\text { temperature saline } \\
\text { solution was used for } \\
5 \text { mins. Small ice } \\
\text { packs were placed } \\
\text { intraorally in the } \\
\text { mouth on the } \\
\text { vestibular surface for } \\
30 \text { minutes } \\
\text { Extraoral } \\
\text { cryotherapy group - } \\
20 \text { mL of room } \\
\text { temperature saline } \\
\text { solution was used }\end{array}$ & $\begin{array}{l}\text { Control group - } \\
20 \mathrm{~mL} \text { room } \\
\text { temperature } \\
\text { saline solution } \\
\text { was used for } \\
5 \text { mins }\end{array}$ & $\begin{array}{l}\text { When compared } \\
\text { with the control } \\
\text { group, all the } \\
\text { cryotherapy groups } \\
\text { exhibited lower } \\
\text { postoperative pain } \\
\text { levels on the first, } \\
\text { third, fifth, and } \\
\text { seventh days and } \\
\text { lower levels of pain } \\
\text { on percussion on } \\
\text { the seventh day }\end{array}$ & $\begin{array}{l}\text { 1st, 3rd, } \\
5 \text { th, 7th } \\
\text { day }\end{array}$ \\
\hline
\end{tabular}




\begin{tabular}{|c|c|c|c|c|c|c|c|c|c|}
\hline Sr No & Author (Year) & $\begin{array}{c}\text { Sample } \\
\text { size }\end{array}$ & $\begin{array}{l}\text { No. of } \\
\text { visit }\end{array}$ & $\begin{array}{l}\text { Type of tooth } \\
\text { /Diagnosis }\end{array}$ & $\begin{array}{c}\text { method of } \\
\text { irrigation used }\end{array}$ & $\begin{array}{l}\text { Final irrigation } \\
\text { regimen in } \\
\text { experimental group }\end{array}$ & $\begin{array}{l}\text { Final irrigation } \\
\text { regimen in } \\
\text { Control group }\end{array}$ & Results & $\begin{array}{l}\text { follow up } \\
\text { (time } \\
\text { interval) }\end{array}$ \\
\hline & & & & & & $\begin{array}{c}\text { for } 5 \text { mins. Ice packs } \\
\text { were placed } \\
\text { extraorally on the } \\
\text { cheek surface for } 30 \\
\text { minutes. }\end{array}$ & & & \\
\hline 6 & $\begin{array}{l}\text { Jain et al. } \\
(2018)^{18}\end{array}$ & 60 & $\begin{array}{l}\text { multiple } \\
\text { visits }\end{array}$ & $\begin{array}{l}\text { mandibular 1st } \\
\text { molar with } \\
\text { symptomatic } \\
\text { irreversible } \\
\text { pulpitis with } \\
\text { symptomatic } \\
\text { apical } \\
\text { periodontitis, } \\
\text { irreversible } \\
\text { pulpitis with } \\
\text { asymptomatic } \\
\text { apical } \\
\text { periodontitis, } \\
\text { irreversible } \\
\text { pulpitis with } \\
\text { normal } \\
\text { periradicular } \\
\text { tissues }\end{array}$ & $\begin{array}{c}28 \text { gauge side } \\
\text { vented needle }\end{array}$ & $\begin{array}{l}\text { Cryotherapy group - } \\
\text { final irrigation was } \\
\text { carried out with } \\
\text { saline at } 2.5^{\circ} \mathrm{C} \text {, using } \\
\text { a } 5 \mathrm{ml} \text { syringe with } \\
28 \text { gauge side vented } \\
\text { needle for a minute. }\end{array}$ & $\begin{array}{l}\text { Control group - } \\
\text { final irrigation } \\
\text { was carried out } \\
\text { with saline at } \\
\text { room } \\
\text { temperature, } \\
\text { using a } 5 \mathrm{ml} \\
\text { syringe with } 28 \\
\text { gauge side } \\
\text { vented needle } \\
\text { for a minute. }\end{array}$ & $\begin{array}{l}\text { At } 6 \text { hours post- } \\
\text { operatively, there } \\
\text { was a statistically } \\
\text { significant reduction } \\
\text { in pain in } \\
\text { experimental group } \\
\text { compared to control } \\
\text { group. At } 24 \text { and } \\
48 \text { hours post- } \\
\text { operatively, there } \\
\text { was no statistically } \\
\text { significant reduction } \\
\text { in pain in } \\
\text { experimental group } \\
\text { compared to control } \\
\text { group. }\end{array}$ & $\begin{array}{c}6,24, \text { and } \\
48 \mathrm{~h}\end{array}$ \\
\hline 7 & $\begin{array}{l}\text { Vieyra et al. } \\
(2019)^{19}\end{array}$ & 240 & $\begin{array}{l}\text { single } \\
\text { visit }\end{array}$ & $\begin{array}{c}\text { maxillary/mandi } \\
\text { bular anterior or } \\
\text { posteriorteeth } \\
\text { with vital teeth } \\
\text { with irreversible } \\
\text { pulpitis }\end{array}$ & Endovac & $\begin{array}{l}\text { Group A - } 5 \mathrm{~mL} \text { of } \\
\text { cold }\left(4^{\circ} \mathrm{C}\right) 17 \% \text { EDTA } \\
\text { followed by } 10 \mathrm{~mL} \text { of } \\
\text { cold }\left(4^{\circ} \mathrm{C}\right) \text { sterile } \\
\text { saline solution } \\
\text { dispensed to the } \mathrm{WL} \\
\left.\text { using a cold ( } 4^{\circ} \mathrm{C}\right) \\
\text { metallic } \\
\text { micro-cannula } \\
\text { included in the Endo } \\
\text { Vac System (Kerr } \\
\text { Endo) and } \\
\text { maintained } \\
\text { intracanally for } 1 \\
\text { minute. } \\
\text { Group B - } 5 \text { mL of } \\
\left.\text { cold ( } 2.5^{\circ} \mathrm{C}\right) 17 \% \\
\text { EDTA followed by } 10 \\
\text { mL of cold }\left(2.5^{\circ} \mathrm{C}\right) \\
\text { sterile saline solution } \\
\text { dispensed to the WL } \\
\text { using a cold }\left(2.5^{\circ} \mathrm{C}\right) \\
\text { metallic } \\
\text { micro-cannula } \\
\text { included in the Endo } \\
\text { Vac System for } 1 \\
\text { minute. }\end{array}$ & $\begin{array}{c}\text { Control group - } 5 \\
\mathrm{~mL} \text { (room } \\
\text { temperature) of } \\
17 \% \text { EDTA } \\
\text { followed by } 10 \\
\mathrm{~mL} \text { (room } \\
\text { temperature) of } \\
\text { sterile saline } \\
\text { solutiondelivered } \\
\text { to the WL using a } \\
\text { metallic } \\
\text { micro-cannulainc } \\
\text { luded in the Endo } \\
\text { Vac System for } 1 \\
\text { minute }\end{array}$ & $\begin{array}{c}\text { No statistically } \\
\text { significant } \\
\text { difference }(\mathrm{P}>0.05) \\
\text { among the groups } \\
\text { was found regarding } \\
\text { degree or duration } \\
\text { of pain. There was } \\
\text { no statistically } \\
\text { significant } \\
\text { difference }(\mathrm{P}>0.05) \\
\text { among the } 40 \mathrm{C} \text { and } \\
2.50 \mathrm{C} \text { groups. }\end{array}$ & $\begin{array}{c}24,48 \text { and } \\
72 \text { hours }\end{array}$ \\
\hline 8 & $\begin{array}{c}\text { Bazaid et al. } \\
(2018)^{20}\end{array}$ & 40 & $\begin{array}{l}\text { multiple } \\
\text { visits }\end{array}$ & $\begin{array}{c}\text { vital teeth, } \\
\text { irreversible } \\
\text { pulpitis with } \\
\text { either normal } \\
\text { apical tissues or } \\
\text { apical } \\
\text { periodontitis }\end{array}$ & $\begin{array}{l}\text { side vented } \\
\text { needles }\end{array}$ & $\begin{array}{l}\text { Group 2: cryotherapy } \\
\text { group ( } n=20 \text { ) } 2.5^{\circ} \mathrm{C} \\
\text { saline was used as } \\
\text { the final irrigant for } \\
2 \text { mins using a side } \\
\text { vented needle Each } \\
\text { group was } \\
\text { subdivided into } 2 \\
\text { subgroups (a \& b } \\
\text { ( } \mathrm{n}=10 \text { ) according to } \\
\text { preoperative apical } \\
\text { diagnosis. a= teeth } \\
\text { with apical } \\
\text { periodontitis b= } \\
\text { teeth with normal } \\
\text { apical tissues. }\end{array}$ & $\begin{array}{l}\text { Group 1: control } \\
\text { group ( } n=20) \\
\text { final irrigant used } \\
\text { was saline at } \\
\text { room } \\
\text { temperature for } \\
2 \text { mins using side } \\
\text { vented needle }\end{array}$ & $\begin{array}{l}\text { A statistically } \\
\text { significant } \\
\text { difference was } \\
\text { found when } \\
\text { comparing the pain } \\
\text { level in cryotherapy } \\
\text { group (subgroup 2a) } \\
\text { with control group } \\
\text { (subgroup 1a) 24, } \\
48 \text { hours } \\
\text { postoperatively. But } \\
\text { there was no } \\
\text { statistically } \\
\text { significant } \\
\text { difference when } \\
\text { comparing the pain } \\
\text { level in cryotherapy }\end{array}$ & $\begin{array}{l}24 \text { hours, } \\
48 \text { hours }\end{array}$ \\
\hline
\end{tabular}




\begin{tabular}{|c|c|c|c|c|c|c|c|c|c|}
\hline Sr No & Author (Year) & $\begin{array}{l}\text { Sample } \\
\text { size }\end{array}$ & $\begin{array}{l}\text { No. of } \\
\text { visit }\end{array}$ & $\begin{array}{l}\text { Type of tooth } \\
\text { /Diagnosis }\end{array}$ & $\begin{array}{c}\text { method of } \\
\text { irrigation used }\end{array}$ & $\begin{array}{l}\text { Final irrigation } \\
\text { regimen in } \\
\text { experimental group }\end{array}$ & $\begin{array}{l}\text { Final irrigation } \\
\text { regimen in } \\
\text { Control group }\end{array}$ & Results & $\begin{array}{l}\text { follow up } \\
\text { (time } \\
\text { interval) }\end{array}$ \\
\hline & & & & & & & & $\begin{array}{c}\text { (subgroup 1b) with } \\
\text { control (subgroup } \\
\text { 1b) after } 24,48 \\
\text { hours } \\
\text { postoperatively }\end{array}$ & \\
\hline 9 & $\begin{array}{l}\text { Dr. Sudheer K. } \\
\text { A et al. } \\
(2019)^{21}\end{array}$ & 60 & $\begin{array}{l}\text { single } \\
\text { visit }\end{array}$ & $\begin{array}{l}\text { Single rooted } \\
\text { vital tooth with } \\
\text { vital teeth, } \\
\text { symptomatic } \\
\text { irreversible } \\
\text { pulpitis }\end{array}$ & $\begin{array}{l}\text { needle and } \\
\text { syringe }\end{array}$ & $\begin{array}{l}\text { Cold saline group - } \\
10 \mathrm{ml} \text { of saline at } \\
\text { about } 2.5^{\circ} \mathrm{C} \text { was used } \\
\text { for final irrigation for } \\
3 \text { minutes }\end{array}$ & $\begin{array}{l}\text { Control group - } \\
10 \mathrm{~mL} \text { of } 0.9 \% \\
\text { physiological } \\
\text { saline solution at } \\
\text { the room } \\
\text { temperature was } \\
\text { used for final } \\
\text { irrigation for } 3 \\
\text { mins }\end{array}$ & $\begin{array}{l}\text { cold saline group } \\
\text { showed a significant } \\
\text { reduction in } \\
\text { postoperative pain } \\
\text { levels in comparison } \\
\text { to control group at } \\
6 \text { to } 24 \mathrm{~h} \text { follow-ups }\end{array}$ & $\begin{array}{l}6,24,48 \\
\text { hours. }\end{array}$ \\
\hline 10 & $\begin{array}{l}\text { Vieyra et al. } \\
(2018)^{22}\end{array}$ & 240 & $\begin{array}{l}\text { single } \\
\text { visit }\end{array}$ & $\begin{array}{l}\text { maxillary/mandi } \\
\text { bular anterior or } \\
\text { posteriorteeth } \\
\text { with vital pulp, } \\
\text { irreversible } \\
\text { pulpitis and no } \\
\text { periapical lesion }\end{array}$ & $\begin{array}{l}\text { endovac } \\
\text { irrigation } \\
\text { system }\end{array}$ & $\begin{array}{l}\text { Group A - } 5 \mathrm{~mL} \text { cold } \\
\left(6^{\circ} \mathrm{C}\right) 17 \% \text { EDTA } \\
\text { followed with } 10 \mathrm{~mL} \\
\text { cold }\left(6^{\circ} \mathrm{C}\right) \text { sterile } \\
\text { saline solution } \\
\text { dispensed to the } \mathrm{WL} \\
\text { using a cold }\left(6^{\circ} \mathrm{C}\right) \\
\text { metallic micro- } \\
\text { cannula included in } \\
\text { the Endo Vac System } \\
\text { (Kerr Endo) and } \\
\text { maintained } \\
\text { intracanally for one } \\
\text { minute. } \\
\text { Group B - } 5 \text { mL cold } \\
\left.\text { (2.5 } 5^{\circ} \mathrm{C}\right) 17 \% \text { EDTA } \\
\text { followed with } 10 \mathrm{~mL} \\
\text { cold }\left(2.5^{\circ} \mathrm{C}\right) \text { sterile } \\
\text { saline solution } \\
\text { dispensed to the WL } \\
\text { using a cold }\left(2.5^{\circ} \mathrm{C}\right) \\
\text { metallic micro- } \\
\text { cannula included in } \\
\text { the Endo Vac System } \\
\text { for one minute. }\end{array}$ & $\begin{array}{c}\text { Group C - final } \\
\text { irrigation with } 5 \\
\text { mL (room } \\
\text { temperature) } \\
17 \% \text { EDTA } \\
\text { followed with } 10 \\
\text { mL (room } \\
\text { temperature) } \\
\text { sterile saline } \\
\text { solution } \\
\text { delivered to the } \\
\text { WLusing a } \\
\text { metallic micro- } \\
\text { cannula included } \\
\text { in the Endo Vac } \\
\text { System forone } \\
\text { minute. }\end{array}$ & $\begin{array}{l}\text { There was no } \\
\text { statistically relevant } \\
\text { difference ( } \mathrm{p}> \\
\text {.05)among Group A } \\
\text { and Group C } \\
\text { compared with } \\
\text { Group B. Group B } \\
\text { showed less pain } \\
\text { than the rest of the } \\
\text { groups }\end{array}$ & $\begin{array}{c}24,48,72 \\
\text { hours }\end{array}$ \\
\hline 11 & $\begin{array}{l}\text { Chauhan et al. } \\
(2020)^{23}\end{array}$ & 40 & $\begin{array}{l}\text { multiple } \\
\text { visits }\end{array}$ & $\begin{array}{l}\text { symptomatic } \\
\text { apical } \\
\text { periodontitis } \\
\text { with pulpal } \\
\text { necrosis }\end{array}$ & $\begin{array}{l}\text { side vented } \\
\text { needles }\end{array}$ & $\begin{array}{l}\text { Group 2: cryotherapy } \\
\text { group }(n=20) \text { where } \\
20 \mathrm{ml} \text { of } 2.5^{\circ} \mathrm{C} \text { saline } \\
\text { was used as the final } \\
\text { irrigant for } 2 \text { mins }\end{array}$ & $\begin{array}{l}\text { Group 1: control } \\
\text { group ( } n=20) \\
\text { where the final } \\
\text { irrigant was } 20 \mathrm{ml} \\
\text { of saline at room } \\
\text { temperature for } \\
2 \text { mins }\end{array}$ & $\begin{array}{l}\text { Patients in the } \\
\text { control group } \\
\text { presented a } \\
\text { significantly higher } \\
\text { incidence of } \\
\text { postoperative pain } \\
\text { intensity, and need } \\
\text { for medication } \\
\text { intake }(\mathrm{P}<.05) \text {. } \\
\text { Cryotherapy } \\
\text { reduced the } \\
\text { incidence of } \\
\text { postoperative pain } \\
\text { and the need for } \\
\text { medication intake. }\end{array}$ & $\begin{array}{l}6,24 \text {, and } \\
72 \text { hours }\end{array}$ \\
\hline 12 & $\begin{array}{c}\text { Vieyra et al. } \\
(2019)^{24}\end{array}$ & 120 & $\begin{array}{l}\text { single } \\
\text { visit }\end{array}$ & $\begin{array}{c}\text { maxillary/mandi } \\
\text { bular anterior or } \\
\text { posteriorteeth } \\
\text { with vital pulp }\end{array}$ & $\begin{array}{l}\text { Endovac } \\
\text { irrigation } \\
\text { system }\end{array}$ & $\begin{array}{l}\text { Group } 6^{\circ} \mathrm{C} \text { - final } \\
\text { irrigation with } 5 \mathrm{~mL} \text { of } \\
\text { cold }\left(6^{\circ} \mathrm{C}\right) 17 \% \text { EDTA } \\
\text { followed by } 10 \mathrm{~mL} \text { of } \\
\text { cold }\left(6^{\circ} \mathrm{C}\right) \text { sterile } \\
\text { saline solution } \\
\text { Group } 4^{\circ} \mathrm{C} \text { - final } \\
\text { irrigation with } 5 \mathrm{~mL} \text { of } \\
\text { cold }\left(4^{\circ} \mathrm{C}\right) 17 \% \mathrm{EDTA} \\
\text { followed by } 10 \mathrm{~mL} \text { of }\end{array}$ & $\begin{array}{l}\text { Group RT - final } \\
\text { irrigation with } \\
5 \mathrm{~mL} \text { (room } \\
\text { temperature) of } \\
17 \% \text { EDTA } \\
\text { followed by } 10 \\
\mathrm{~mL} \text { (room } \\
\text { temperature) of } \\
\text { sterile saline } \\
\text { solution }\end{array}$ & $\begin{array}{c}\text { No statistically } \\
\text { major change } \\
\text { between the groups } \\
\text { was found regarding } \\
\text { the degree or } \\
\text { duration of pain. }\end{array}$ & $\begin{array}{c}24,48,72 \\
\text { hours }\end{array}$ \\
\hline
\end{tabular}




\begin{tabular}{|c|c|c|c|c|c|c|c|c|c|}
\hline Sr No & Author (Year) & $\begin{array}{c}\text { Sample } \\
\text { size }\end{array}$ & $\begin{array}{l}\text { No. of } \\
\text { visit }\end{array}$ & $\begin{array}{l}\text { Type of tooth } \\
\text { /Diagnosis }\end{array}$ & $\begin{array}{l}\text { method of } \\
\text { irrigation used }\end{array}$ & $\begin{array}{l}\text { Final irrigation } \\
\text { regimen in } \\
\text { experimental group }\end{array}$ & $\begin{array}{l}\text { Final irrigation } \\
\text { regimen in } \\
\text { Control group }\end{array}$ & Results & $\begin{array}{l}\text { follow up } \\
\text { (time } \\
\text { interval) }\end{array}$ \\
\hline & & & & & & $\begin{array}{l}\text { cold }\left(4^{\circ} \mathrm{C}\right) \text { sterile } \\
\text { salinesolution }\end{array}$ & & & \\
\hline 13 & $\begin{array}{c}\text { Alharthi et al. } \\
(2019)^{25}\end{array}$ & 105 & $\begin{array}{l}\text { single } \\
\text { visit }\end{array}$ & $\begin{array}{c}\text { single rooted } \\
\text { teeth, diagnosis } \\
\text { not mentioned }\end{array}$ & $\begin{array}{l}\text { two side- } \\
\text { vented needle } \\
\text { with gauge } \\
\text { size } 30\end{array}$ & $\begin{array}{c}\text { Cryotherapy group - } \\
10 \mathrm{~mL} \text { of cold (1.5- } \\
\left.2.5^{\circ} \mathrm{C}\right) 0.9 \% \text { normal } \\
\text { saline solution was } \\
\text { used for } 5 \text { mins } \\
\text { Room temperature } \\
\text { saline group }-10 \mathrm{~mL} \\
\text { of room-temperature } \\
0.9 \% \text { normal saline } \\
\text { solution was used for } \\
5 \text { mins }\end{array}$ & $\begin{array}{l}\text { Control group- } \\
\text { received no more } \\
\text { irrigation. }\end{array}$ & $\begin{array}{l}\text { There was no } \\
\text { significance } \\
\text { difference between } \\
\text { Cryotherapy group } \\
\text { and Room } \\
\text { temperature saline } \\
\text { group. The highest } \\
\text { post-endodontic } \\
\text { pain was in control } \\
\text { group. } \\
\text { In general, the post- } \\
\text { endodontic pain (6 } \\
\text { h, } 24 \mathrm{~h} \text {, and } 48 \\
\text { h)decreased with } \\
\text { time in all groups. }\end{array}$ & $\begin{array}{c}6,24 \text {, and } \\
48 \mathrm{~h}\end{array}$ \\
\hline
\end{tabular}

\section{Risk of bias in individual studies}

Risk of bias was assessed by the two independent reviewers for RCTs included in the review and discrepancies were resolved by discussion and appropriate consultation with a third reviewer. The domains for risk assessment were graded as low, uncertain or high risk, based on selection bias (random sequence generation and allocation concealment), performance bias (blinding), attrition bias (incomplete outcome data), detection bias (assessor blinding) and reporting bias (selective reporting). Thus, the overall risk for individual studies were assessed as low, moderate or high risk based on the domains and criteria.

\section{RESULTS}

\section{Data analysis}

The PRISMA guidelines were followed for the methodology. The study selection process is summarized in Figure. 1 (PRISMA flow chart). All the titles and abstracts were screened based on the stringent selection criteria. Subsequently the full texts were assessed independently by the two reviewers. A total of thirteen studies over the past five years met the inclusion criteria for full text reading and all thirteen were included for further analysis.

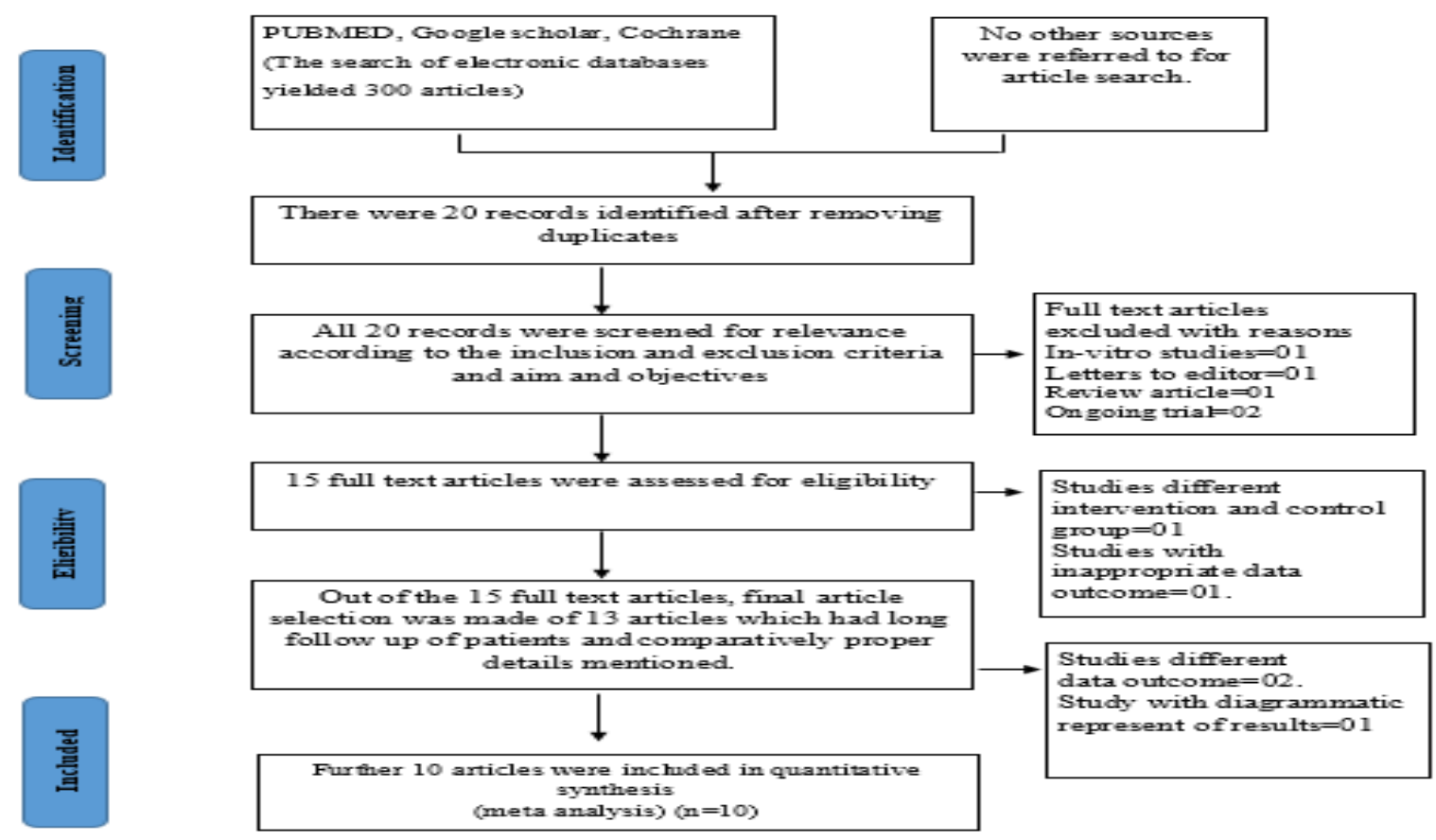

Figure 1: PICOS Search Strategy Flowchart 


\section{Study characteristics}

Thirteen articles were selected for qualitative synthesis and ten articles $14,15,16,17,18,19,22,23,24,25$ for quantitative synthesis. The characteristics of all the included studies are listed in Table 1. All the studies were in-vivo clinical trials performed on adult patients with age ranging from 18-65 years. Two studies ${ }^{15,16}$ did not mention the age of the participants. Pre-operative pulpal status assessment of these studies showed that eleven studies included teeth with irreversible pulpitis ${ }^{13-23}$, of which four studies ${ }^{19,21,22,24}$ included teeth indicated for intentional root canal treatment for prosthetic reason. Preoperative periapical status assessment revealed seven studies ${ }^{14-18,20,23}$ included teeth with both apical periodontitis or normal periapical status, while one study did not mention the pulpal or periapical diagnosis ${ }^{25}$. Root canal treatments were performed in a single visit in eight studies ${ }^{13,14,17,19,21,22,24,25}$ and in two visits in the other five studies ${ }^{15,16,18,20,23}$. In two visit endodontic treatment postoperative endodontic pain evaluation was done at the end of the first appointment. Assessment of the tooth type revealed four studies ${ }^{14,19,22,24}$ included both multirooted and single rooted teeth, while one study ${ }^{17}$ included only molars and one study included only mandibular fist molars ${ }^{18}$. Three studies ${ }^{16,20,23}$ did not mention the type of tooth while four studies included only single rooted teeth ${ }^{13,15,21,25}$. Final irrigation was performed using cold saline at a temperature range of $1.5^{\circ} \mathrm{C}-6^{\circ} \mathrm{C}$ with $2.5^{\circ} \mathrm{C}$ being the most commonly used temperature. Three studies used cold EDTA in the final irrigation regimen followed by the use of cold saline ${ }^{19,22,24}$. The method of irrigation assessment revealed that conventional needle and syringe was used for irrigation in eight studies ${ }^{14,16-}$ $18,20,21,23,25$ while five studies used sterile microcannula attached to negative pressure irrigation system (Endovac) $^{13,15,19,22,24}$ The final rinse volume ranged from 5 $\mathrm{mL}^{14,18}, 10 \mathrm{~mL}^{19,22,24,25}$ to $20 \mathrm{~mL}^{13,15-17,23}$ and duration of use ranged between $1 \min ^{18,19,22,24}$ and $5 \min ^{13-17,25}$. Follow up of postoperative pain ranged from 6hrs to 7days and was recorded using a visual analogue scale (VAS) ${ }^{13-23,25}$. Seven studies evaluated preoperative pain levels ${ }^{14-}$ $17,20,21,23$, one study excluded patients with preoperative pain $^{25}$ and five studies did not evaluate preoperative pain $^{13,18,19,22,24}$.

The meta-analysis was conducted on ten studies $14,15,16,17,18,19,22,23,24,25$ which have qualified with required data outcome that could be analysed quantitatively. The other studies were excluded as the data reported could not be analysed (which was not in mean+/sd format). The results are depicted as forest plot in Figure.2. We considered heterogeneity significant if the $\mathrm{I}^{2}$ values were above $50 \%$.(Cochrane collaboration). With the meta-analysis conducted for the selected studies, the heterogeneity was insignificant $\mathrm{I}^{2}=89 \%$, hence we applied the random effect model. The mean difference was -0.26 ($0.49,-0.03)$ thus indicating that the mean VAS score experience was more in control group than the cryotherapy intervention.

Further a subgroup analysis for the different time intervals at which pain was assessed was conducted. With the metaanalysis conducted for the selected studies at 6 hours $\left(1^{2}=\right.$ $85 \%, M D=-1.15(-2.15,-0.15), p=0.02)$ [Figure $3 \mathrm{~A}$ ] and $24 \mathrm{hrs}\left(\mathrm{I}^{2}=91 \%, \mathrm{MD}=-0.39(-0.79,0.01), \mathrm{p}=0.05\right)$ [Figure. $3 \mathrm{~B}]$ the mean VAS score experience was more in control group than the cryotherapy intervention. While at $48 \mathrm{hrs}\left(\mathrm{I}^{2}\right.$ $=09 \%, \mathrm{MD}=-0.02(-0.08,0.03), \mathrm{p}=0.41)$ [Figure. $3 \mathrm{C}$ ] and $72 \mathrm{hrs}\left(\mathrm{I}^{2}=88 \%, \mathrm{MD}=-0.14(-0.49,0.21), \mathrm{p}=0.43\right)$ [Figure. $3 D$ ] there was no significant reduction in postoperative pain.

\section{Risk of bias}

The methodological quality of all the studies was done using the risk-of-bias assessment tool as elaborated in Cochrane Handbook for Systematic Review of Interventions (version 5.1.0). The results are presented in [Figure. $4 \mathrm{~A}$ and $\mathrm{B}$ ] as the risk of bias graph and summary respectively which was generated using the RevMan software (v5.3). There are six domains under which the methodology of individual studies is assessed and granted a level of risk. The quality assessment of included twelve studies was done with representation [Figure. $4 \mathrm{~A}$ and $\mathrm{B}$ ]. One study ${ }^{16}$ (Jaiswal et al) was excluded for assessment due to the contradiction in the study design. All the studies showed either unclear allocation concealment or no mention of blinding of participants or the outcome assessed which was not possible due to the study design. All the studies had moderate level of methodology overall that could be followed and none had a high risk of level of quality.

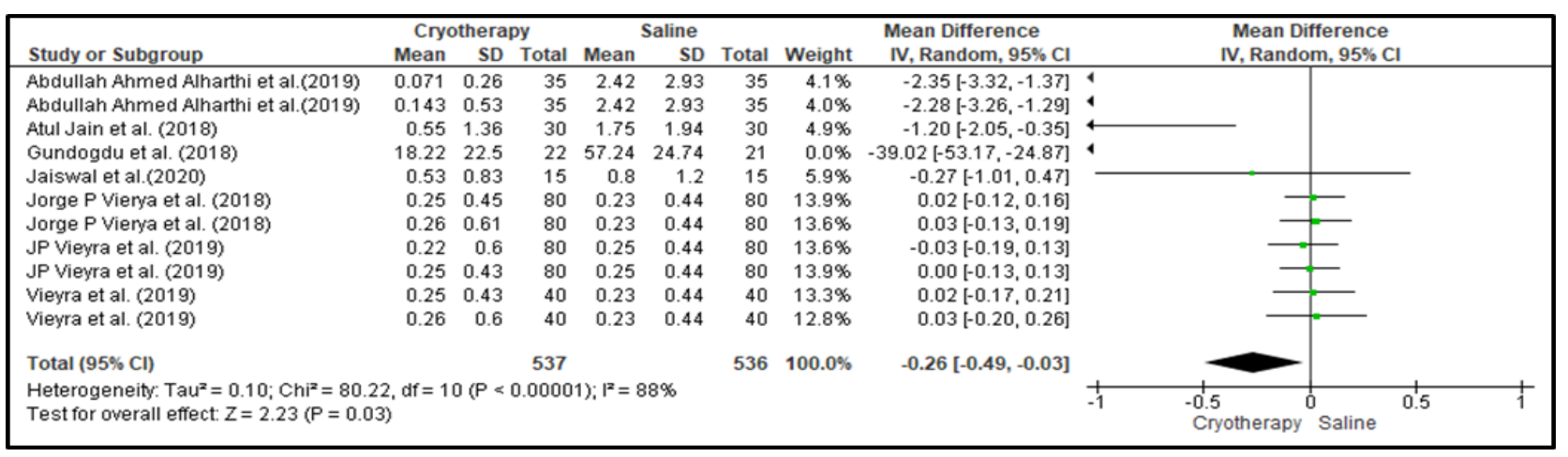

Figure 2: Forest plot 


\section{A. Postoperative pain at 6 hours}

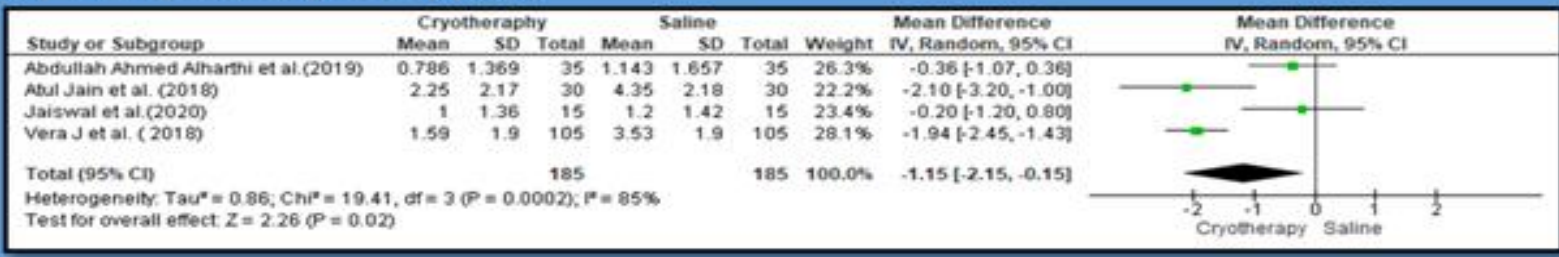

\section{B. Postoperative pain at $\mathbf{2 4}$ hours}

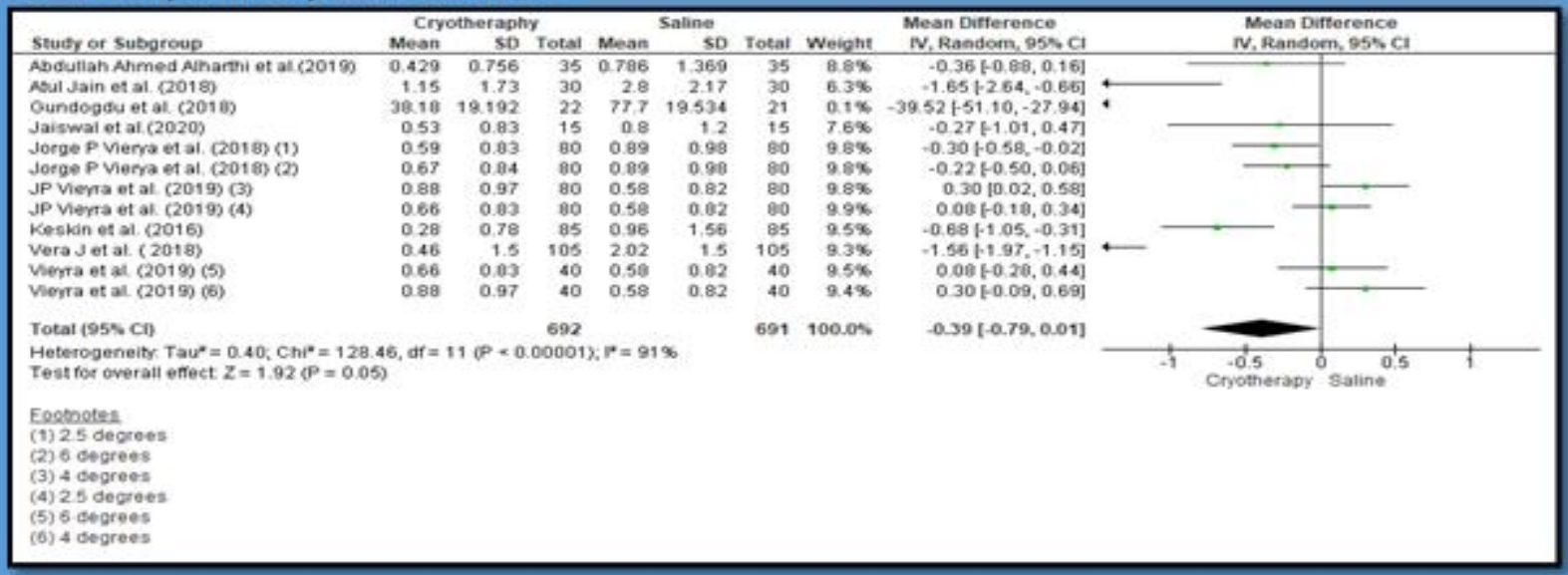

\section{Postoperative pain at $\mathbf{4 8}$ hours}

\begin{tabular}{|c|c|c|c|c|c|c|c|c|c|c|c|c|}
\hline \multirow[b]{2}{*}{ Study or Subgroup } & \multicolumn{3}{|c|}{ Cryotheraphy } & \multicolumn{3}{|c|}{ Saline } & \multicolumn{3}{|c|}{ Mean Difference } & \multirow{2}{*}{\multicolumn{3}{|c|}{ Mean Difference }} \\
\hline & Meas & So & Total & Mean & so & Total & Weight & iv, Fíced, 95s Ca & & & & \\
\hline Abdullah Ahmed Alharthi et at (2019) & 0.071 & 0.267 & 35 & 0.143 & 0.535 & 35 & $7.8 \%$ & $-0.07[-0.27,0.13]$ & & & & \\
\hline Ahut Jain ef al. (2018) & 0.55 & 1.36 & 30 & 1.75 & 1.94 & 30 & $0.4 \%$ & $-1.20+2.05,-0.35$ & $\leftarrow$ & & & \\
\hline Jorge P Vierya et al $(2018)$ (1) & 0.23 & 0.44 & 80 & 0.26 & 0.61 & 80 & $11.2 \%$ & $-0.03\{-0.19,0.13\}$ & & & & \\
\hline Jorge P Vierya en at (2018) (2) & 0.25 & 0.45 & 800 & 0.26 & 0.61 & 80 & $11.0 \%$ & $-0.01[-0.18,0.16]$ & & & & \\
\hline JP Vieyra ot al. (2019) (3) & 0.25 & 0.43 & 80 & 0.23 & 0.44 & 80 & $16.7 \%$ & $0.02[-0.11,0.15]$ & & & & \\
\hline JP Viegra et al. $(2019)$ (4) & 0.26 & 0.8 & 80 & 0.23 & 0.44 & 80 & $11,4 \%$ & $0.03[-0.13,0.19]$ & & & & \\
\hline Keskin et al. (2016) & 0.02 & 0.21 & 85 & 0.07 & 0.45 & 85 & $27.3 \%$ & $-0.05 ;-0.16,0.06\}$ & & 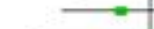 & - & \\
\hline Vierra et al (2019) (5) & 0.254 & 0.43 & 40 & 0.25 & 0.44 & 40 & $8.4 \%$ & $0.00+0.19,0.19$ & & & & \\
\hline Vierra et at $(2019)(B)$ & 0.22 & 0.8 & 40 & 0.25 & 0.44 & 40 & $5.7 \%$ & $.0 .0310 .26,0.201$ & & & & \\
\hline Total $(95 \mathrm{sl})$ & & & 550 & & & 550 & 100.05 & $-0.02[-0.08,0.03]$ & & & & \\
\hline $\begin{array}{l}\text { Heterogenend } \mathrm{ChP}=8.80, \mathrm{df}=8(\mathrm{P}= \\
\text { Test for overall effect } Z=0.83(\mathrm{P}=0.4\end{array}$ & 1) 36$) ; P^{P}=$ & & & & & & & & & $\begin{array}{l}-0.2=0.1 \\
\text { Cryotherapy }\end{array}$ & Saline & \\
\hline Eeonctes: & & & & & & & & & & & & \\
\hline (1) 2.5 degrees & & & & & & & & & & & & \\
\hline (2) 5 degrees & & & & & & & & & & & & \\
\hline (3) 4 degrees & & & & & & & & & & & & \\
\hline $\begin{array}{l}\text { (4) } 2.5 \text { degrees } \\
\text { (5) } 5 \text { deorees }\end{array}$ & & & & & & & & & & & & \\
\hline $\begin{array}{l}\text { (5) } 6 \text { degrees } \\
\text { (6) } 4 \text { degrees }\end{array}$ & & & & & & & & & & & & \\
\hline
\end{tabular}

\section{Postoperative pain at 72 hours}

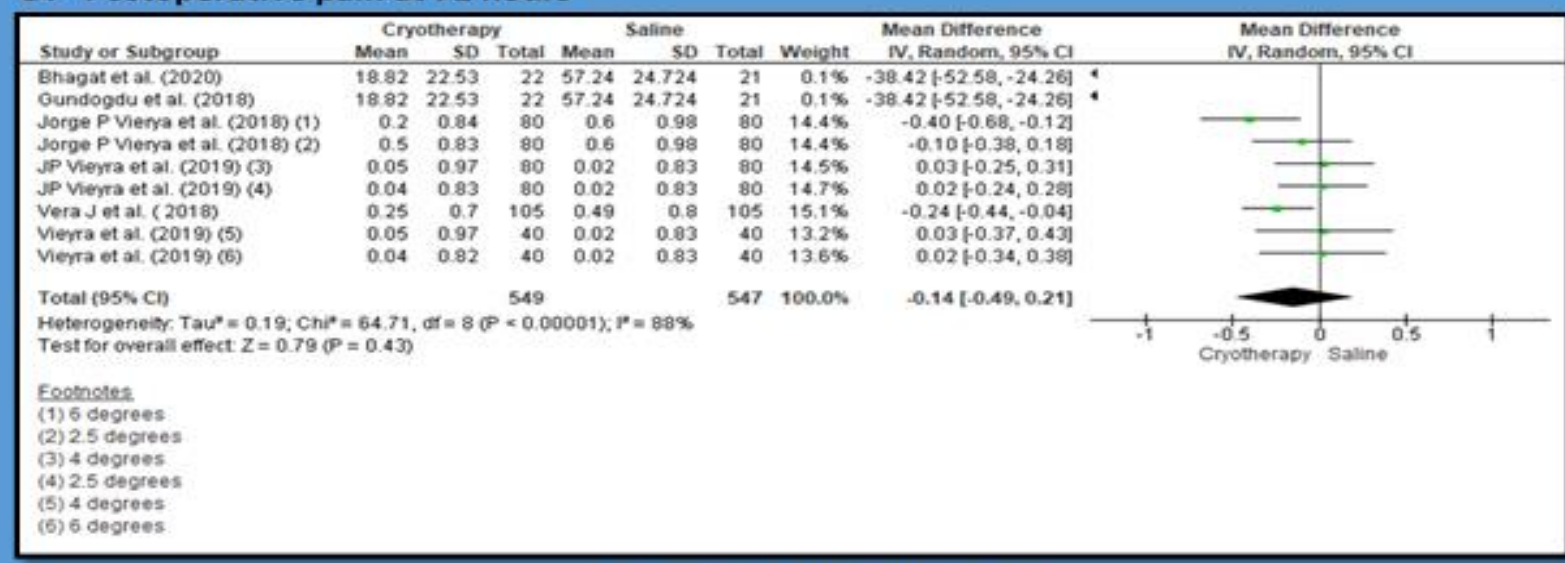

FIGURE 3: Subgroup analysis 


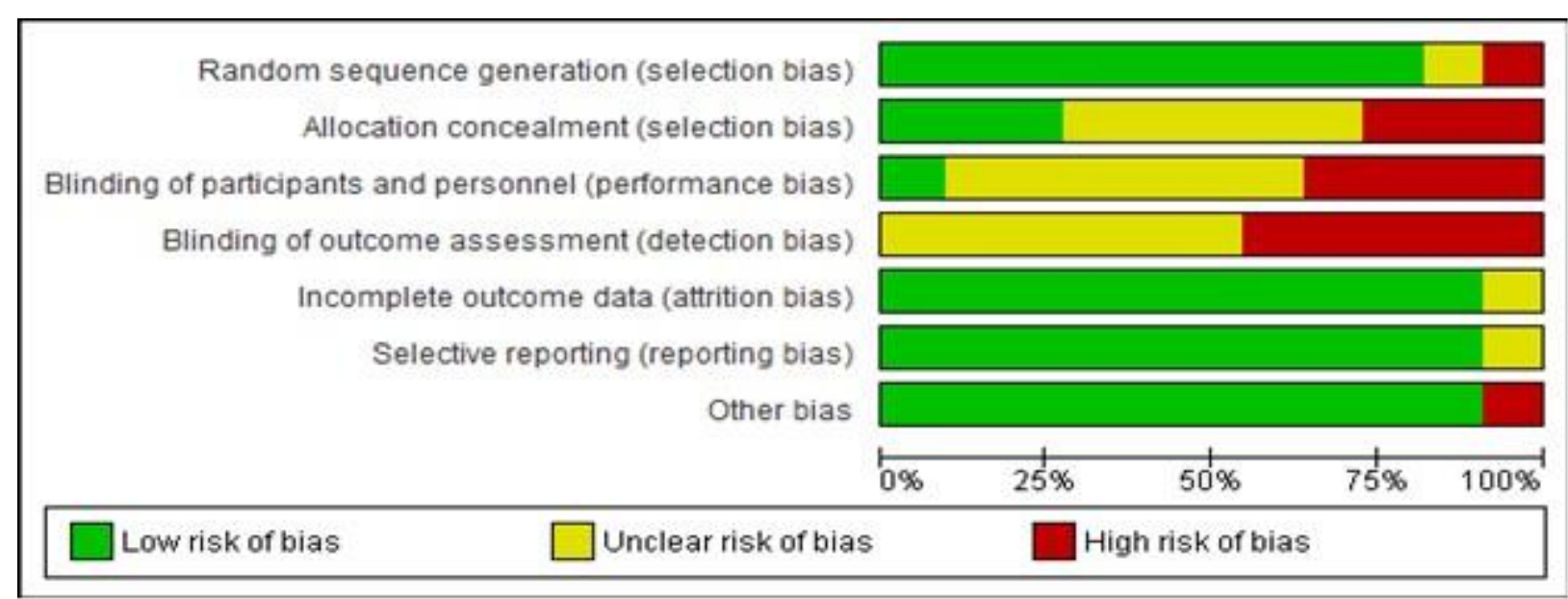

\section{A. Risk of Bias Assessment}

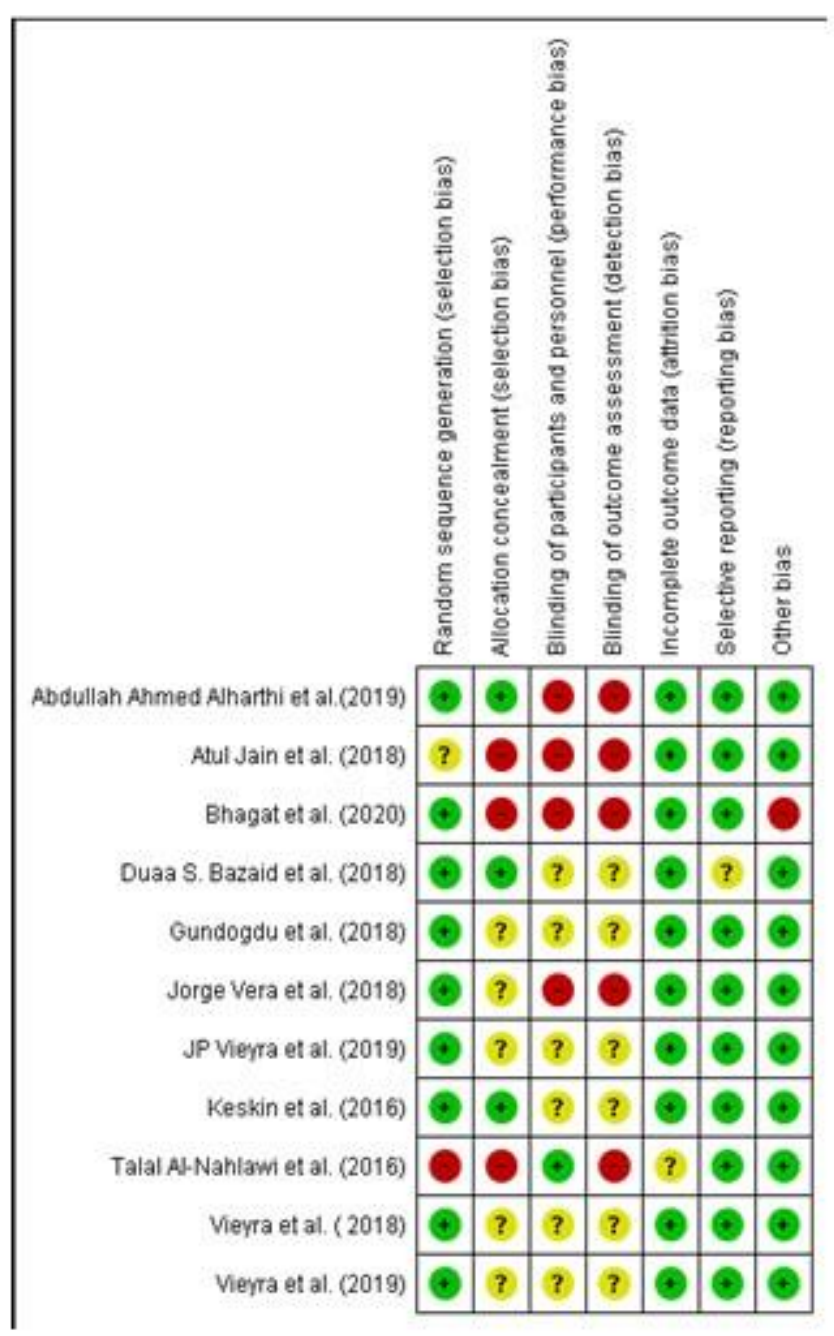

\section{B. Risk Assessment Table}

Figure 4: Risk of Bias Assessment

\section{DISCUSSION}

Necrotic pulps usually provide an environmental condition that is conducive to the establishment of several different oral bacterial species, particularly strictly anaerobic bacteria $^{3}$. Among the studies included in this systematic review only three studies ${ }^{15,16,23}$ included teeth with necrotic pulp while teeth with vital pulp were included in other studies ${ }^{13,17,19-22,24}$. Positive relationship between necrotic teeth with painful apical pathosis and flare-up rate was found in most of the studies ${ }^{26,27}$.

Also, cases with pre-existing periapical pathology have a higher possibility of exacerbation of pre-existing periapical inflammation which could be attributed to the passage of minor quantity of irritants and microorganisms into the 
periapical region ${ }^{28}$. Out of the thirteen articles reviewed, seven articles ${ }^{14-18,20,23}$ have included teeth with periapical pathology while five articles ${ }^{13,19,21,24}$ have included teeth with no periapical pathology. Six ${ }^{14,15,17,18,20,23}$ out of seven studies which included cases with pre-existing periapical inflammation (apical periodontitis) reported reduction in postoperative pain due to the effect of intracanal cryotherapy while one study by Jaiswal et al. ${ }^{16}$ reported no significant difference in the postoperative pain with the use of intracanal cryotherapy.

In a randomized multicentre clinical trial by Vera et al. ${ }^{15}$, cryotherapy was shown to reduce both the incidence of postoperative pain and the need for medication in patients presenting with a diagnosis of necrotic pulp and symptomatic apical periodontitis ${ }^{15}$ Results of the studies which evaluated the effect of cryotherapy in reducing postoperative pain in cases of irreversible pulpitis with and without apical periodontitis ${ }^{18,20}$ showed that cryotherapy was effective only in patients diagnosed with apical periodontitis, whereas in patients with only irreversible pulpitis, there was no significant difference in the incidence of postoperative pain between the groups ${ }^{20}$. This could be attributed to the anti -inflammatory effect of cryotherapy which is produced due to the reduction in the temperature, caused by the use of cold saline resulting in decrease in the periapical tissue edema and inflammation ${ }^{20}$.

With the increase in preference of single visit endodontics by both the dentist and patient owing to its efficiency and convenience, effectiveness of intracanal cryotherapy in reducing postoperative pain in single visit endodontic treatment should be evaluated. However, the importance of multivisit endodontic treatment in case of necrotic, nonvital teeth with periapical pathology or retreatment cannot be completely overviewed. Hence, it is important to evaluate the effectiveness of intracanal cryotherapy in reducing postoperative pain in both single visit and multivisit endodontics. Recent studies ${ }^{13,14,17,21,23}$ that evaluated the effect of cryotherapy on single-visit root canal treatment (RCT), showed a reduction in the pain levels as compared to the control group, while four other studies $^{19,22,24,25}$ showed no significant difference between the groups. Multivisit endodontics were performed in four studies $^{15,16,18,20}$ in which three studies ${ }^{15,18,20}$ showed reduction in postoperative pain after the use of intracanal cryotherapy.

Inclusion of both single and multirooted teeth in studies $^{14,19,22,24}$ may also affect the results of the study due to noncategorization of the variables resulting in comparison between teeth with different anatomical configurations. Hence, considering all the above mentioned factors, single rooted anterior teeth and multirooted posterior teeth need to be evaluated separately and cannot be considered at par with each other.

Use of negative apical pressure irrigation by Al Nahlawi et al. ${ }^{13}$ in conjunction to intracanal cryotherapy showed a reduction in post-obturation pain at 6 hours, when compared with control group where Endovac system was not used. This could be attributed to the less irritation and more efficient cleaning of the EndoVac system ${ }^{29}$. Thus, four studies ${ }^{13,15,19,22}$ included in this review did not include the conventional technique of irrigation and instead have used EndoVac system across all the groups. On the contrary other studies ${ }^{14,17,18,20,23}$ using conventional needle and syringe for irrigation, showed a decrease in postoperative pain in cryotherapy group compared to the control group.

A strong positive correlation is seen between preoperative pain and postoperative pain values, where preoperative pain scores significantly influences the immediate postoperative pain score ${ }^{5,7}$. This could be explained by the preoperative presence of an infected root canal system and/or periapical region, which, may become secondarily irritated during treatment resulting in postoperative pain $^{26}$. Seven studies ${ }^{14-17,20,21,23}$ included in this review evaluated preoperative pain and found intervention to be effective.

Cryotherapy is derived from Greek words "cryos" denoting "cold" and "therapeia" denoting "cure" ${ }^{30}$. It is a form of treatment in which the body is briefly exposed to very cold temperatures in order to bring about healing and other therapeutic results. Cryotherapy can be applied to the inflamed periradicular tissues by irrigating agents like EDTA or saline at lower temperature, as final irrigating agents $^{21}$. Cold EDTA was used in three studies ${ }^{19,22,24}$ followed by application of cold saline while ten other studies $^{13-18,20,21,23,25}$ used only cold saline as the final irrigating agent. Irrigating agents were used at a lower temperature range of $1.5^{\circ} \mathrm{C}-6^{\circ} \mathrm{C}$, with $2.5^{\circ} \mathrm{C}$ being the most commonly used temperature. This can be attributed to the findings of Franz and $\operatorname{lggo}^{31}$ which stated that lowering the body temperature decreases peripheral nerve conduction, and in particular, when it reaches about $7^{\circ} \mathrm{C}$, there is complete deactivation of myelinated $A-\delta$ fibres, whereas deactivation of nonmyelinated C-fibre occurs at about $3^{\circ} \mathrm{C}$. Lower temperatures used in cryotherapy also triggers thermal receptors, which on stimulation blocks nociception within the spinal cord and as a result reduces the transmission of painful stimuli. Vera et al. ${ }^{15}$ in his in vitro study demonstrated a reduction in the external root surface temperature by more than $10^{\circ} \mathrm{C}$ for 4 minutes with the use of $2.5^{\circ} \mathrm{C}$ saline solution as the final irrigating agent. This decrease in temperature is said to produce local anti-inflammatory effect which is beneficial to inflamed periradicular tissues. An optimal dosage for cryotherapy has not been determined; the two studies ${ }^{14,18}$ included in this review used a volume of $5 \mathrm{ml}$, while five studies $^{19,21,22,24,25}$ used $10 \mathrm{ml}$ and five studies ${ }^{13,15-17,23}$ used $20 \mathrm{ml}$ of cold irrigating solution over a duration of 2-5 mins.

Postoperative pain most often occurs during the first 2448 hours after obturation, and generally recedes in a few hours, although it occasionally persists for several days ${ }^{5}$. Thus any strategy used for reduction of postoperative pain 
should be most effective during the first 24-48 hours. Cryotherapy has been found to be effective in reducing postoperative pain at $6 \mathrm{hrs}^{13,15,18,21,23}, 24 \mathrm{hrs}{ }^{13-15,17,18,20,21,23}$ , 48hrs ${ }^{13,14,20,21}$ and $72 \mathrm{hrs}^{15,17,23}$. This can be attributed to the three basic physiologic tissue responses -decrease in metabolic activity, blood flow and inhibition in neural receptors. Drop in local temperature is the first physiologic tissue response to cryotherapy, resulting in reduced cellular metabolism. This causes cells to use less oxygen and reduces blood flow as induced by vasoconstriction, which produces an antiedema effect and, hence, a consequent reduction of inflammation ${ }^{32}$.

Meta-analysis was conducted for the eligible studies which accounted for ten ${ }^{14,15,16,17,18,19,22,23,24,25}$ studies in which the data outcome was analyzed quantatively. Outcomes were extracted based on VAS score as the pain parameter. The heterogeneity was higher hence random effect model was applied and the cumulative mean difference was derived. The mean difference showed that the VAS score was higher in the control group than the cryotherapy group. This indicated the effectiveness of intervention of intracanal cryotherapy in reducing pain during endodontic therapy rather than just using saline at room temperature. The temperature of solution used during irrigation showed variation at $2^{\circ} \mathrm{C}, 4^{\circ} \mathrm{C}$ and $6^{\circ} \mathrm{C}$. The use and effect of cryotherapy was thus proved statistically effective in our systematic review and meta-analysis.

\section{Limitations}

The variability among the studies with respect to the method of application of intracanal cryotherapy, such as the use of different irrigation techniques, the different temperatures and volumes used could be seen as a limitation. Direct extrapolation of the dependent and independent variables in assessment of post-endodontic pain which is multifactorial in nature may not be possible in randomized clinical trials. Technical difficulties in maintaining the temperature of the irrigant have neither been specified nor addressed.

\section{Future studies}

Effect of intracanal cryotherapy on the physiological healing process of periapical tissues and the duration required for complete healing to occur needs to be further evaluated. Standardizing should be taken into consideration in order to eliminate potential confounding factors and allow the analysis of these factors individually. Also, further studies need to be undertaken to identify an optimal dosage, duration and volume of intracanal cryotherapy.

\section{CONCLUSION}

Within the limitations of the study, it can be concluded that intracanal cryotherapy using cold saline or $17 \%$ EDTA at a temperature range of $2.5^{\circ} \mathrm{C}$ to $6^{\circ} \mathrm{C}$ as final irrigating agent helps in reducing postoperative pain at $6 \mathrm{hrs}$ and 24 hrs following single-visit or multi visit root canal treatment in teeth diagnosed with irreversible pulpitis with apical periodontitis. Thus, intracanal cryotherapy can be suggested as a simple, cost-effective, and non-toxic method for management of postoperative pain following endodontic treatment.

\section{REFERENCES}

1. Pak JG, White SN. Pain Prevalence and Severity before, during, and after Root Canal Treatment: A Systematic Review. J Endod. 2011 Apr; 37(4): 429-38.

2. Sathorn C, Parashos $P$, Messer $H$. The prevalence of postoperative pain and flare-up in single- and multiple-visit endodontic treatment: a systematic review. Int Endod J. 2008; 41(2): 91-9.

3. Siqueira JF. Microbial causes of endodontic flare-ups. Int Endod J. 2003; 36(7): 453-63.

4. EIMubarak AH, Abu-bakr NH, Ibrahim YE. Postoperative pain in multiple-visit and single-visit root canal treatment. Journal of endodontics. 2010 Jan 1; 36(1): 36-9.

5. Alí A, Olivieri JG, Duran-Sindreu F, Abella F, Roig M, GarcíaFont $M$. Influence of preoperative pain intensity on postoperative pain after root canal treatment: A prospective clinical study. J Dent. 2016 Feb; 45: 39-42.

6. Gatchel RJ. Dealing with discomfort managing anxiety and pain during dental treatment. J Am Dent Assoc. 1992; 123(6): 37-41.

7. Parirokh M, Rekabi AR, Ashouri R, Nakhaee N, Abbott PV, Gorjestani H. Effect of occlusal reduction on postoperative pain in teeth with irreversible pulpitis and mild tenderness to percussion. J Endod. 2013; 39(1): 1-5.

8. Arslan H, Gündoğdu EC, Sümbüllü $M$. The effect of preoperative administration of antihistamine, analgesic and placebo on postoperative pain in teeth with symptomatic apical periodontitis: a randomized controlled trial. Eur Endod J. 2016; 1(1): 1.

9. Attar S, Bowles WR, Baisden MK, Hodges JS, McClanahan SB. Evaluation of pretreatment analgesia and endodontic treatment for postoperative endodontic pain. J Endod. 2008; 34(6): 652-5.

10. Gallatin E, Reader A, Nist R, Beck M. Pain reduction in untreated irreversible pulpitis using an intraosseous injection of Depo-Medrol. J Endod. 2000; 26(11): 633-8.

11. Laureano F JR, e SILVA ED de O, CAMARGO IB, Gouveia FM. The influence of cryotherapy on reduction of swelling, pain and trismus after third-molar extraction: a preliminary study. J Am Dent Assoc. 2005; 136(6): 774-8.

12. Kullenberg B, Ylipää S, Söderlund K, Resch S. Postoperative cryotherapy after total knee arthroplasty: a prospective study of 86 patients. J Arthroplasty. 2006; 21(8): 1175-9.

13. Al-Nahlawi T, Hatab TA, Alrazak MA, Al-Abdullah A. Effect of Intracanal Cryotherapy and Negative Irrigation Technique on Postendodontic Pain. J Contemp Dent Pract. 2016 Dec; 17(12): 990-6.

14. Keskin C, Özdemir Ö, Uzun İ, Güler B. Effect of intracanal cryotherapy on pain after single-visit root canal treatment. Aust Endod J. 2017 Aug; 43(2): 83-8.

15. Vera J, Ochoa J, Romero M, Vazquez-Carcaño M, RamosGregorio CO, Aguilar RR, et al. Intracanal Cryotherapy 
Reduces Postoperative Pain in Teeth with Symptomatic Apical Periodontitis: A Randomized Multicenter Clinical Trial. J Endod. 2018 Jan; 44(1): 4-8.

16. Jaiswal S, Vagarali H, Pujar M, Kapshe N. Effect of cold saline irrigation on postoperative pain-A randomized control trial.

17. Gundogdu EC, Arslan H. Effects of Various Cryotherapy Applications on Postoperative Pain in Molar Teeth with Symptomatic Apical Periodontitis: A Preliminary Randomized Prospective Clinical Trial. J Endod. 2018 Mar; 44(3): 349-54.

18. Jain A, Davis D, Bahuguna R, Agrawal A, Singh $S$, Ramachandran $\mathrm{R}$, et al. Role of cryotherapy in reducing postoperative pain in patients with irreversible pulpitis. an in-vivo study. Int J Den Med Sci. 2018; 2: 43-9.

19. Vieyra JP, Enriquez FJJ, Acosta FO, Guardado JA. Reduction of postendodontic pain after one-visit root canal treatment using three irrigating regimens with different temperature. Niger J Clin Pract. 2019; 22(1): 18-24.

20. Bazaid DS, Kenawi LMM. The Effect of Intracanal Cryotherapy in Reducing Postoperative Pain in Patients with Irreversible Pulpitis: A Randomized Control Trial. Int J Health Sci. 2018; (2): 6.

21. Sudheer KA, Idris $M$, Geeta IB, et al. Effect on post endodontic pain after single setting root canal treatment in vital tooth following final irrigation with cold saline. Int J Sci Res 2019; 8: 41-3.

22. Vieyra JP and Guardado JA. Reduction of Post-Endodontic Pain after One-Visit Root Canal Treatment Using Three Cryotherapy Protocols with Different Temperature. Ann Materials Sci Eng. 2018; 3(2): 1033.

23. Chauhan K, Bhushan J. Efficacy of Intracanal Cryotherapy in Reducing Post Operative Pain in Patients with Symptomatic
Apical Periodontitis: An In-Vivo Study. J Adv Med Dent Sci Res. 2020; 8(9): 6-11.

24. Vieyra JP, Guardado JA, Casillas FC, Vargas AH. Post Endodontic Pain Reduction using three Irrigants with Different Temperature. Sch J Oto 2019; 2(2): SJO. MS.ID.000134

25. Alharthi AA, Aljoudi MH, Almaliki MN, Almalki MA, Sunbul MA. Effect of intra-canal cryotherapy on post-endodontic pain in single-visit RCT: A randomized controlled trial. Saudi Dent J. 2019 Jul; 31(3): 330-5.

26. Mathew ST. Post operative pain in endodontics: A systemic review. J Dent Oral Hyg. 2015; 7(8): 130-7.

27. Alamassi BY. Endodontic postoperative pain: etiology and related factors-an update. Int J Dent Sci Res. 2017; 5(2): $13-21$.

28. Sindhu S, Nadig RR, Pai VS, Nair S. Endodontic Flare UpsAn Overview. Int J Contemp Med Res. 2015; 2(2).

29. Mancini M, Cerroni L, lorio L, Armellin E, Conte G, Cianconi L. Smear layer removal and canal cleanliness using different irrigation systems (EndoActivator, EndoVac, and passive ultrasonic irrigation): field emission scanning electron microscopic evaluation in an in vitro study. J Endod. 2013; 39(11): 1456-60.

30. Fayyad DM, Abdelsalam N, Hashem N. Cryotherapy: A New Paradigm of Treatment in Endodontics. J Endod. 2020 Jul; 46(7): 936-42.

31. Franz DN, Iggo A. Conduction failure in myelinated and non-myelinated axons at low temperatures. J Physiol. 1968; 199(2): 319-45.

32. Capps SG, Brook M. Cryotherapy and intermittent pneumatic compression for soft tissue trauma. Int J Athl Ther Train. 2009; 14(1): 2-4.

Source of Support: The author(s) received no financial support for the research, authorship, and/or publication of this article.

Conflict of Interest: The author(s) declared no potential conflicts of interest with respect to the research, authorship, and/or publication of this article.

For any question relates to this article, please reach us at: globalresearchonline@rediffmail.com New manuscripts for publication can be submitted at: submit@globalresearchonline.net and submit_ijpsrr@rediffmail.com 